\title{
ASPECTOS RELACIONADOS À UTILIZAÇÃO DA EQUAÇÃO LOGÍSTICA QUADRÁTICA EM PROCESSOS ELETROQUÍMICOS
}

\author{
Hamilton Varela \\ Fritz-Haber-Institut der Max-Planck-Gesellschaft, Faradayweg 4-6, D-14195 Berlin, Germany. \\ Roberto M. Torresi e Ernesto R. Gonzalez* \\ Instituto de Química de São Carlos, Universidade de São Paulo, CP 780, 13560-970 São Carlos -SP
}

Recebido em 10/10/00; aceito em 7/5/01

\begin{abstract}
ASPECTS RELATED TO THE USE OF THE QUADRATIC LOGISTIC EQUATION IN ELECTROCHEMICAL PROCESSES. The concepts of dissipation and feedback are contained in the behavior of many natural dynamical systems. They have been used to predict the evolution of populations leading to the formulation of the quadratic logistic equation (QLE). More recently, the QLE has been used to provide a better understanding of physicochemical systems with promising results. Many physical, chemical and biological dynamic phenomena can be understood on the basis of the QLE and this work describes the main aspects of this equation and some recent applications, with emphasis on electrochemical systems. Also, it is illustrated the concept of potential energy as a convenient way of describing the stability of the fixed points of the QLE.
\end{abstract}

Keywords:logistic equation; intercalation process; electrochemical systems; feedback.

\section{INTRODUÇÃO}

Um dos problemas centrais em áreas como biologia, demografia e ecologia humana está relacionado à capacidade do ambiente em proporcionar condições de sobrevivência à determinada espécie. Nesse aspecto, Thomas Robert Malthus(1766 - 1834) foi o primeiro a desenvolver uma teoria sobre o crescimento populacional humano relacionando-o à capacidade do ambiente em prover esse crescimento $^{1}$. Sobre a importância do trabalho de Malthus pode-se citar, por exemplo, sua influência no desenvolvimento do conceito de seleção natural por Darwin, que representa o ponto de partida para toda toda a biologia evolucionária moderna e ecologia ${ }^{2}$. A idéia malthusiana do crescimento incontrolável de população sujeito apenas a restrições ambientais foi colocada em termos matemáticos por Verhulst ${ }^{3}$, que utilizou a equação de crescimento logístico no aumento populacional da França, Bélgica, Rússia e Inglaterra em 1838. Desde então, a equação de crescimento logístico ou equação logística quadrática (ELQ) vem sendo utilizada em modelos matemáticos aplicados a ecologia (principalmente na descrição de processos de crescimento populacional), relações presa-predador, interações competitivas, gerenciamento de fontes renováveis, evolução da resistência a pesticidas, controle ecológico de pestes, entre outros ${ }^{4}$.

Considerando que características como não-linearidade, dissipação e retro-alimentação (feedback) em sistemas dinâmicos estão contidas na ELQ, sua utilização vem sendo recentemente descrita para a modelagem de processos físico-químicos como resposta de células a combustível com eletrólito polimérico ${ }^{5,6}$, transporte de cargas em filmes orgânicos- e inorgânicos ${ }^{8}$ finos. Outro exemplo bastante ilustrativo da utilização da ELQ por analogia com sistemas biológicos trata da criação sucessiva de defeitos, como o processo de fotodegradação em semicondutores amorfos ${ }^{10}$. Particularmente considerando as aplicações de filmes eletroativos em, por exemplo, baterias secundárias, dispositivos eletrocrômicos e sensores ${ }^{11-16} \mathrm{se}$ fazem necessárias investigações a respeito dos mecanismos de transporte nesses filmes. Uma das maiores dificuldades na utilização da

* e-mail: ernesto@iqsc.sc.usp.br
ELQ em sistemas eletroquímicos como os exemplificados acima reside exatamente no tratamento relativo à utilização de conceitos de sistemas não-lineares e modelos dinâmicos.

O objetivo desse trabalho é descrever a utilização da ELQ em processos eletroquímicos influenciados por retro-alimentação e que apresentam características não-lineares. A abordagem utilizada foi escolhida com o objetivo de enfatizar os conceitos de dinâmica não-linear envolvidos, no sentido de facilitar a compreensão da linguagem utilizada na discussão dos exemplos. Obviamente optou-se apenas por introduzir o jargão em detrimento à descrição matemática exaustiva dos conceitos envolvidos, no entanto, para leitores interessados nesse tema, sugerimos a consulta de literatura especializada ${ }^{17-20}$.

\section{CONCEITOS FUNDAMENTAIS}

O ponto de partida na descrição do sistema dinâmico em estudo é a evolução temporal de uma determinada população. Assim, considerando $\mathrm{x}(\mathrm{t})$ como sendo a população de espécies no tempo $t$, a taxa de variação dessa população pode ser dada como,

$$
\frac{\mathrm{dx}}{\mathrm{dt}}=\text { nascimentos-mortes+migração }
$$

a qual representa simplesmente uma equação de conservação para a população. A forma dos termos do lado direito da equação depende das condições específicas do modelo a ser considerado. O modelo mais simples não considera o termo relacionado à migração e os outros dois termos (mortes e nascimentos) são proporcionais a $\mathrm{x}$, de modo que a relação (1) pode ser escrita como

$$
\frac{d x}{d t}=b x-d x=(b-d) x=\text { const } x
$$

cuja solução é dada por,

$$
x(t)=x_{0} e^{(b-d) t}
$$

na qual, $b$ e $d$ são constantes positivas e $\mathrm{x}(0)=\mathrm{x}_{0}$ é a população inicial. Portanto, se $b>d$, a população cresce exponencialmente, enquanto se $b<d$, a população diminui também de forma exponencial. Em 
linhas gerais, esse modelo é considerado não realista para descrever o sistema representado pela equação (1) já que não inclui nenhuma restrição ao crescimento exponencial ilimitado. Portanto, para contornar essa dificuldade, um termo que represente uma restrição ao crescimento populacional deve ser incluído. A inclusão de um processo autolimitante operando quando a população se torna muito grande conduz a equação:

$$
\frac{\mathrm{dx}}{\mathrm{dt}}=\operatorname{rx}\left(1-\frac{\mathrm{x}}{\mathrm{K}}\right)
$$

com $r$ e $K$ sendo constantes positivas. Tal forma é conhecida como crescimento logístico e a equação como a equação logística quadrática (ELQ). Nesse modelo, a taxa de nascimento per capita é dada pelo termo $\mathrm{r}(1-\mathrm{x} / \mathrm{K})$ e depende de $x$. A constante $K$ está relacionada à capacidade do ambiente de suprir o aumento populacional e é usualmente determinada pelas fontes de sustentação do ambiente. Observe-se que o termo (1-x/K) na equação (4) se opõe ao crescimento temporal de $\mathrm{x}$. Este fenômeno é conhecido como "retro-alimentação" e será discutido posteriormente com mais detalhes utilizando um modelo químico.

A solução da equação (4) é dada por

$$
x(t)=\frac{x_{0} K^{r t}}{\left[K+x_{0}\left(e^{r t}-1\right)\right]}
$$

$\mathrm{Na}$ qual, o termo $\mathrm{x}_{0}=\mathrm{x}(0)$ representa a população inicial. Para tempos muito longos $(\mathrm{t} \rightarrow \infty)$ a população tende ao valor constante $K$. A Figura 1 ilustra o comportamento de $\mathrm{x}(\mathrm{t})$ em função do tempo para valores significativos da população inicial $\mathrm{x}_{0}$.

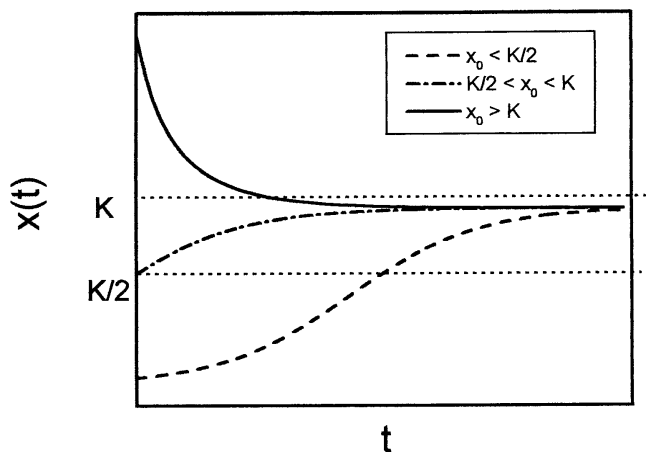

Figura 1. Dependência da variável generalizada x com o tempo t de acordo com a aquação (5) para diferentes populações iniciais $\left(x_{0}=0,07 ; 1,26\right.$ e 4; para $\mathrm{K}=2$ ).

Para valores iniciais, $\mathrm{x}_{0}$, maiores que $\mathrm{K}, \mathrm{x}(\mathrm{t})$ decresce monotonicamente até $\mathrm{K}$, enquanto que para valores iniciais menores que $K, x(t)$ cresce até $K$. No segundo caso $\left(x_{0}<K\right)$ há uma diferença qualitativa de comportamento quando $\mathrm{x}_{0}<\mathrm{K} / 2$ e o perfil de $\mathrm{x}(\mathrm{t})$ em função do tempo apresenta um formato sigmoidal. À primeira vista pode-se encarar a situação em que $\mathrm{x}_{0}>\mathrm{K}$ como implicando em uma taxa negativa de nascimento per capita, o que, obviamente, não tem sentido físico. Na realidade, o resultado ilustra que as taxas de nascimento e imigração somadas são menores que a soma das taxas de morte e emigração, como mostrado na equação (1).

A solução analítica da equação $(4)$ (fazendo $\mathrm{dx} / \mathrm{dt}=0$ ) fornece como raízes 0 e $\mathrm{K}$, esses valores são denominados pontos fixos e representados pelo símbolo $\mathrm{x}$ * Pontos fixos são classificados como estáveis e instáveis de acordo com a análise da estabilidade linear, ou seja, através da linearização em torno do ponto fixo ${ }^{17}$. Caso uma perturbação imposta ao sistema cresça em torno do ponto fixo, esse é considerado instável; caso a perturbação decresça, o ponto fixo em questão é assinalado como sendo estável. Tal análise corresponde à observação do comportamento da primeira derivada da função $f(x)$ no(s) ponto(s) fixo(s). Para o caso da equação (2), tem-se que $\mathrm{f}^{\prime}(0)=\mathrm{r}(\mathrm{e}$ portanto, $>0)$ e $\mathrm{f}^{\prime}(\mathrm{K})=-\mathrm{r}(<0)$, assim, o ponto $\mathrm{x}^{*}=0$ é instável e $\mathrm{x}^{*}=\mathrm{K}$ estável. O gráfico de dx/dt em função de x é mostrado na Figura 2, tal representação é conhecida como o diagrama ou plano de fase do sistema e ilustra a disposição dos pontos fixos $x^{*}=0$ e $x^{*}=K$.

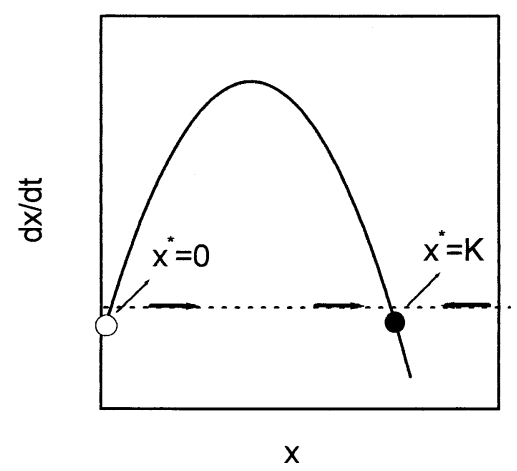

Figura 2. Esquema da dependência de dx/dt com $x$ de acordo com a forma da ELQ dada na equação (4).

Em termos da equação diferencial original, pontos fixos representam soluções de equilíbrio (estacionárias, constantes ou de repouso $)^{21}$. O estado de equilíbrio é dito ser estável se todas as peturbações suficientes pequenas são assimiladas pelo sistema e destruídas no tempo. Portanto, o equilíbrio estável é representado geometricamente por pontos fixos estáveis. Por outro lado, na condição de equilíbrio instável operante, a perturbação cresce no tempo e tal equilíbrio é representado por pontos fixos instáveis. Na Figura 2 o ponto fixo instável $x^{*}=0$ é representado por um círculo vazio e o ponto fixo estável $\mathrm{x}^{*}=\mathrm{K}$ por um círculo cheio e os sentidos das setas no eixo $\mathrm{x}$ representam o sentido do crescimento, ou seja, enquanto o ponto fixo instável repele as trajetórias a partir deste, o ponto fixo estável atrai essas trajetórias. Por esse motivo, pontos fixos instáveis e estáveis são também chamados de repulsores e atratores, respectivamente. Assim, o significado físico do comportamento de dx/dt ilustrado na Figura 2 é dado a seguir. A partir do valor inicial $\mathrm{x}_{0}$, o sistema se afasta com uma certa aceleração positiva do ponto fixo instável e atinge o máximo em $\mathrm{x}=\mathrm{K} / 2$, após esse ponto de máximo o sistema se aproxima do ponto fixo estável com uma aceleração negativa. Em termos de população, observa-se que o crescimento populacional tende para o valor de $\mathrm{K}$, como discutido acima. Obviamente, se o valor inicial $\mathrm{x}_{0}$ for um dos dois pontos fixos $(0 \mathrm{ou} \mathrm{K})$, a população permanece constante.

Em sistemas de primeira ordem $(\mathrm{dx} / \mathrm{dt}=\mathrm{f}(\mathrm{x}))$ como o mostrado na ELQ, a dinâmica pode ser visualizada através do conceito de energia potencial. No contexto deste conceito, a função $f(x)$ estaria relacionada com um potencial $V(x)$ de forma tal que $f(x)=-d V / d x$, sendo que o sinal negativo segue a convenção usual em física de que o sistema evolui para o estado de menor energia potencial. Para um ponto fixo no sistema de primeira ordem $\mathrm{dx} / \mathrm{dt}=0$. Como $\mathrm{dV} / \mathrm{dt}=$ $\mathrm{dV} / \mathrm{dx} . \mathrm{dx} / \mathrm{dt}=-(\mathrm{dV} / \mathrm{dx})^{2}<0$ o ponto fixo corresponde a situação na qual $\mathrm{x}$ não varia com o tempo e, portanto, $\mathrm{dV} / \mathrm{dx}=0$, ou seja que $\mathrm{V}$ permanece constante. Em outras palavras, um ponto fixo do sistema dinâmico $(\mathrm{dx} / \mathrm{dt}=0)$ corresponde a um máximo (se o ponto fixo for estável) ou a um mínimo (se o ponto fixo for instável) do campo vetorial definido por $\mathrm{V}(\mathrm{x})$.

Retornando ao caso da análise da estabilidade dos pontos fixos da ELQ, o potencial para a equação (2) mostrada anteriormente é obtido a partir da expressão $d x / d t=-d V / d x=r x(1-x / K)$ e assume a seguinte forma, 


$$
\mathrm{V}(\mathrm{x})=-\frac{\mathrm{rx}^{2}}{2}+\frac{\mathrm{rx}^{3}}{3 \mathrm{~K}}=-\mathrm{rx}^{2}\left(\frac{1}{2}-\frac{\mathrm{x}}{3 \mathrm{~K}}\right)
$$

na qual a constante de integração foi tomada como zero por conveniência.

A Figura 3 ilustra a correspondência entre os pontos fixos da equação (4) e a curva de potencial calculada com a equação (6). Em ambos os pontos fixos 0 e $\mathrm{K}$ fica claro o que foi dito a respeito da estabilidade desses pontos. $\mathrm{O}$ primeiro ponto fixo, $\mathrm{x}=0$, é instável e corresponde a um máximo na curva de potencial, por outro lado, o segundo ponto fixo é estável e corresponde a um mínimo na curva de potencial. Nesse ponto, vale salientar que modelos mais elaborados devem incluir condições específicas do sistema em estudo no sentido de tornar a descrição do processo o mais realística possível, com formas alternativas da ELQ ${ }^{21-24}$.

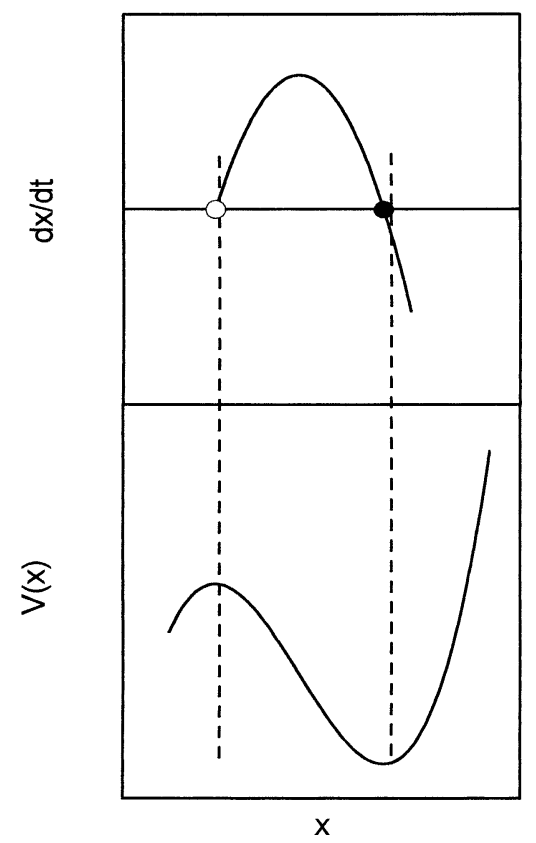

Figura 3. Gráficos de dx/dt (equação (4)) e V(x) (equação (6)) em função de $x$, mostrando a correlação entre os pontos fixos da ELQ e o máximo e mínimo da função potencial.

O termo de retro-alimentação relacionado à ELQ pode ser melhor discutido através de um esquema semelhante ao de uma reação química com mecanismo autocatalítico generalizado:

$$
\mathrm{A}+\mathrm{X} \stackrel{\mathrm{r}}{\longrightarrow} 2 \mathrm{X}
$$

$\mathrm{Na}$ analogia com sistemas biológicos utilizada, $X$ representa os indivíduos e $A$ as condições vitais necessárias ao desenvolvimento. Assim, a multiplicação das espécies $X$ é influenciada pela sua população na forma de retro-alimentação positiva ${ }^{28}$. Em reações químicas, tal mecanismo é um dos responsáveis pelas oscilações no modelo de Lotka-Volterra ${ }^{26,27}$, considerando a participação de outras espécies.

A taxa de produção de $X$ é dada por,

$$
\frac{\mathrm{dx}}{\mathrm{dt}}=\mathrm{rxa}
$$

na qual $a$ e $x$ denotam as concentrações de $A$ e $X$, respectivamente. Uma distinção adicional considerando a equação (7) deve ser feita com relação a concentração da espécie $A$. Caso a concentração de $A$ seja mantida constante, tem-se a situação caraterizada pelo crescimento ilimitado da espécie $X$, tal fenômeno é conhecido como "autocatálise explosiva". Por outro lado, caso a concentração de $A$ decresça durante a reação autocatalítica tem-se o processo denominado "autocatálise autolimitante". Em outras palavras, a retro-alimentação positiva está contida em $X$ e representa o termo de ativação. O termo $A$, por sua vez, relacionase à re-alimentação negativa que representa a inibição ao aumento de $X$. Com relação aos sistemas biológicos ilustrados, a primeira situação ("autocatálise explosiva") conduziria a um aumento explosivo da espécie $X$ graças às fontes ilimitadas de condições de vida (como alimentação e espaço físico, por exemplo), enquanto no segundo caso ("autocatálise autolimitante") um termo relativo às limitações ao processo de reprodução é incluído na equação (8) através da concentração de $A$. O equacionamento para o processo autolimitante é dado considerando como constante a soma dos termos $A$ e $\quad X(\mathrm{~A}+\mathrm{X}=\mathrm{cte})^{28}$, dessa forma, a concentração de $A$ na equação (8) é dada em termos de $x$ como sendo,

$$
\mathrm{a}=1-\frac{\mathrm{x}}{\mathrm{K}}
$$

Na qual, a constante $K$ é a mesma definida anteriormente e normaliza a concentração de $x$. Obviamente, a substituição da equação (9) na (8) conduz à formulação inicial da ELQ mostrada na equação (4).

Através do que foi dito, o sistema de re-alimentação descrito na ELQ e dado em termos do mecanismo de autocatálise autolimitante deve ser interpretado considerando dois mecanismos atuantes: realimentação positiva (através da autocatálise direta, equação 7) e realimentação negativa (devido ao consumo de $A$, segundo o mecanismo regulatório na equação 9$)^{29}$.

\section{SISTEMAS ELETROQUÍMICOS}

"Understanding what is going on arround us is equivalent to building models and confronting them with observations. This statement may sound like a truism to a physicist or chemist, but it goes far beyond physics and chemistry. At each moment our sensory systems scan the surroundings, the brain registers and compares the observations with respect to images already formed, and eventually reaches a preliminary conclusion. One of the basic steps in this procedure is the extensive use of analogies and archetypes."

G. Nicolis e Y. Prigogine ${ }^{30}$

Nesta seção serão ilustrados alguns exemplos da utilização da ELQ em processos eletroquímicos. A principal característica a ser explorada em tais sistemas está primariamente associada ao comportamento das propriedades estudadas em função de sua derivada com respeito ao tempo. Assim, em todos os casos será discutido o comportamento dessas variáveis e comparado ao mecanismo de crescimento/diminuição de dx/dt em função de x segundo a ELQ como mostrada acima.

Uma diferença a ser ressaltada em relação aos trabalhos anteriores utilizando a $\mathrm{ELQ}^{5-9}$ reside na sua formulação: nesses trabalhos utilizou-se a forma normalizada $\mathrm{dx} / \mathrm{dt}=\mathrm{ax}(1-\mathrm{x})$, (na qual, $0 \leq \mathrm{x} \leq 1$ ) enquanto na presente descrição optou-se pela forma mostrada na equação (4) em que dois parâmetros ( $r$ e $K$ ) são utilizados. De fato, essa formulação não introduz diferenças adicionais na análise, já que as características não lineares de crescimento/diminuição do termo $\mathrm{dx} /$ dt são equivalentes nas duas formas. A vantagem da utilização da ELQ na forma dada pela equação (2) está no fato de permitir normalizar os termos $\mathrm{dx} / \mathrm{dt}$ e $\mathrm{x}$ de forma independente. Como será mostrado mais adiante, os gráficos comparativos utilizando a ELQ serão dados de forma adimensional, já que o interesse é observar o comportamento qualitativo descrito pela propriedade $x$, e ordenadas e abcissas estão normalizadas. 
Finalmente, deve ser ressaltado que a característica relativa ao crescimento espontâneo de população a partir de um pequeno valor inicial discutido para sistemas vivos encontra seu correspondente no gradiente de potencial gerado, por exemplo, pela variação do potencial (entre os eletrodos de trabalho e referência, via a utilização de um potenciostato) em sistemas eletroquímicos e, portanto a condição inicial de valor zero para a propriedade em estudo não está relacionada à condição de equilíbrio discutida para "sistemas vivos". assim, como em sistemas vivos o sistema não se afasta do primeiro ponto fixo quando a população é zero, em nossos exemplos o gradiente gerado promove o afastamento desse ponto inicial.

\section{A Potência de Células à Combustível}

O primeiro trabalho tratando de sistemas eletroquímicos utilizando a ELQ foi publicado em 1996 por Gonzalez ${ }^{5}$. Nesse trabalho o autor utilizou a ELQ na discussão dos resultados de densidade de potência de células à combustível com eletrólito polimérico em função da diferença de potencial. Assim curvas de potência (ou produto da diferença de potencial e corrente) em função da diferença de potencial foram racionalizadas em termos da ELQ.

$\mathrm{O}$ conceito envolvido nesse primeiro trabalho considera o fluxo de carga através de uma célula a combustível como sendo um processo dissipativo e realimentado. A potência da célula é definida por $\mathrm{P}=\mathrm{dE} / \mathrm{dt}$, onde $\mathrm{E}$ é a energia, e o potencial da célula $(\mathrm{V})$ pode ser equiparado à energia por unidade de carga. Assim, a ELQ normalizada neste caso pode ser escrita $\mathrm{dE} / \mathrm{dt}=4 \mathrm{E}(1-\mathrm{E})$ ou, de forma equivalente, $\mathrm{P}=4 \mathrm{~V}(1-\mathrm{V})$. Curvas de potência em função do potencial, normalizadas, podem ser comparadas com a ELQ, como mostrado na Figura 4. Os desvios observados a potenciais maiores são devidos ao fato de que nessa região o fluxo de carga é controlado pela cinética de transferência de carga através da interface e não pelos fenômenos de dissipação e realimentação.

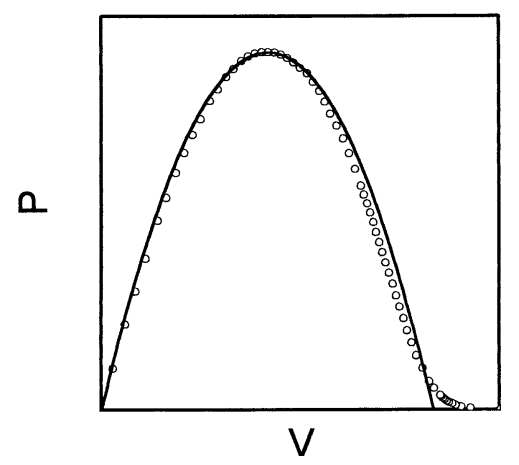

Figura 4. Comparação entre a potência experimental normalizada de uma célula a combustível (símbolos) com a ELQ normalizada (curva contínua).

Uma interpretação mais detalhada deste sistema foi feita posteriormente, utilizando um modelo microscópico que separa os diferentes efeitos que contribuem à queda de potencial com o aumento da corrente $^{6}$. A análise demonstrou que os efeitos que contribuem para o resultado mostrado na Figura 4 são aqueles efetivamente controlados por dissipação e realimentação.

\section{Transporte em Filmes Inorgânicos Finos}

O segundo exemplo a ser discutido trata do comportamento eletrocrômico de filmes finos de $\mathrm{Ni}(\mathrm{OH})_{2}$. Torresi et al. ${ }^{8}$ discutiram as variações de absorbância em filmes de $\mathrm{Ni}(\mathrm{OH})_{2}$ durante o processo redox em condições potenciodinâmicas em solução aquosa de
KOH. Assim, a transição redox $\mathrm{Ni}^{2+} / \mathrm{Ni}^{3+}$ foi monitorada através da corrente e da absorbância. A Figura 5 ilustra o comportamento redox desses filmes através de perfis de corrente ( $\mathrm{j}$, ou fluxo de carga, dq/ dt) e da derivada da absorbância em relação ao tempo (dA/dt) em função do potencial. Na comparação desses dois perfis, observa-se que ambos apresentam picos de oxidação e redução bem definidos em praticamente os mesmos valores de potencial, indicando que a carga correspondente a ambos os processos de oxidação e redução está basicamente relacionada às transformações cromogênicas no interior do filme em praticamente toda a faixa de potencial estudada. No entanto, em potenciais anódicos acima de $0,35 \mathrm{~V}$ observa-se que a quantidade $\mathrm{dA} / \mathrm{dt}$ não reflete o perfil observado em $\mathrm{j}$, o que é atribuído à parcela de corrente consumida na reação de geração de oxigênio (RGO). De fato, os autores encontraram que cerca de $30 \%$ da carga consumida no processo anódico está relacionada a $\mathrm{RGO}^{8}$.

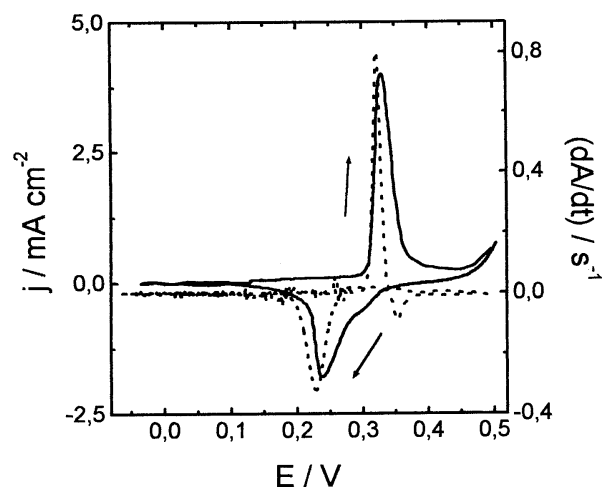

Figura 5. Comportamento redox de filmes finos de $\mathrm{Ni}(\mathrm{OH})_{2}$, expresso através das dependências com o potencial da corrente $(j=d q / d t$, -$)$ e da derivada da absorbância (dA/dt, -------).

No sentido de avaliar a relação entre os fluxos de espécies responsáveis pela manutenção da eletroneutralidade no interior do filme durante o processo redox, estes foram estudados em função da carga, q, e da absorbância, A, de forma direta através do termo dA/dt. Na Figura 6 são mostradas as taxas dA/dt em função de A durante os processos de oxidação e de redução, assim como as curvas representando a ELQ. A excelente correlação observada corrobora com a hipótese inicial do transporte regido pela lei não-linear na forma da ELQ, no qual o pico no perfil dA/dt versus A reflete a presença de uma separatriz entre as etapas de crescimento e diminuição do fluxo das espécies através da interface filme/solução. Nesse ponto vale salientar o papel de técnicas in situ como espectroeletroquímica no estudo das propriedades de transporte para esse sistema específico, já que participação de cátions, ânions e moléculas de solvente durante o processo tornam uma análise via microbalança eletroquímica a cristal de quartzo um tanto difícil ${ }^{31}$. Assim, reações paralelas como a RGO não produzem variações na absorbância e a análise de como essa propriedade varia durante o processo redox, fornece informações diretas sobre o transporte de espécies, sem a necessidade de levar em conta a contribuição de outros $\operatorname{processos}^{32,33}$.

\section{Transporte em Filmes Poliméricos Finos}

$\mathrm{Na}$ avaliação das propriedades eletroquímicas dos polímeros condutores eletrônicos deve se considerar o processo de transição entre os estados isolante e condutor que ocorre em função do transporte de espécies ditas dopantes através da matriz polimérica. O estudo da natureza dessas espécies dopantes assim como do seu meca- 


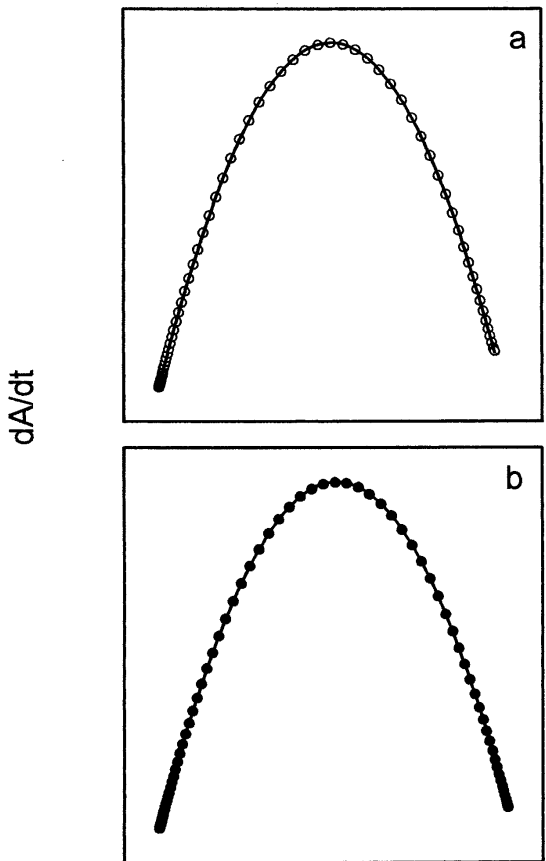

A

Figura 6. Comparação da dependência de dA/dt com A (normalizadas) com a forma normalizada da ELQ para filmes finos de $\mathrm{Ni}(\mathrm{OH})_{2}$. (a) oxidação e (b) redução.

nismo de transporte assumem importância capital na avaliação das potenciais aplicações desses materiais ${ }^{34}$. Uma discussão sobre o transporte em eletrodos poliméricos modificados pode ser encontrada na literatura recente ${ }^{35}$. Particularmente a utilização da microbalança eletroquímica a cristal de quartzo (MECQ) ${ }^{36-39}$ associada a medidas eletroquímicas tradicionais fornece importantes informações sobre as espécies que participam do processo de compensação de cargas e sobre o transporte dessas espécies que cruzam a interface filme/solução através de medidas de variação de massa in situ.

Como descrito previamente ${ }^{40-42}$, através dos dados de corrente e variação de massa obtidos durante experimentos simultâneos de voltametria cíclica e MECQ é possível obter os fluxos de cátions e ânions mais a contribuição do solvente a partir das equações de variação de massa e carga segundo as condições de eletroneutralidade no interior do filme. Tais fluxos são dados em função da densidade de corrente e do fluxo de massa, nas equações,

$$
\frac{\mathrm{d}\left(\xi_{\mathrm{C}^{+}(\mathrm{E})}+\alpha \xi_{\mathrm{SE})}\right)}{\mathrm{dt}}=\frac{1}{\mathrm{~W}_{\mathrm{CA}}} \frac{\mathrm{d}\left(\Delta \mathrm{m}_{(\mathrm{E})}\right)}{\mathrm{dt}}+\frac{\mathrm{W}_{\mathrm{A}^{-}}}{\mathrm{W}_{\mathrm{CA}}} \frac{\mathrm{j}_{\mathrm{EE}}}{\mathrm{F}}
$$

para cátions, e,

$$
\frac{\mathrm{d}\left(\xi_{\mathrm{A}^{-}(\mathrm{E})}+\alpha \xi_{\mathrm{SE})}\right)}{\mathrm{dt}}=\frac{1}{\mathrm{~W}_{\mathrm{CA}}} \frac{\mathrm{d}\left(\Delta \mathrm{m}_{(\mathrm{E})}\right)}{\mathrm{dt}}+\frac{\mathrm{W}_{\mathrm{C}^{+}}}{\mathrm{W}_{\mathrm{CA}}} \frac{\mathrm{j}_{\mathrm{EE})}}{\mathrm{F}}
$$

para ânions. Nessas equações, F é a constante de Faraday, W é a massa molar de cátions $\left(\mathrm{C}^{+}\right)$, ânions $\left(\mathrm{A}^{-}\right)$ou moléculas de solvente (S), $\xi$ é a quantidade de cátions, ânions ou moléculas de solvente, $\alpha=\mathrm{W}_{\mathrm{S}} / \mathrm{W}_{\mathrm{CA}}$ e CA corresponde ao sal utilizado no eletrólito. $\mathrm{O}$ subíndice $(\mathrm{E})$ indica que a magnitude depende do potencial do eletrodo. Nas equações (10) e (11), fluxos negativos se referem à ejeção e positivos à incorporação de espécies no filme e ambos os fluxos contém uma parcela referente à participação de solvente já que o conjunto de equações iniciais (balanços de carga e massa) permanece indeterminado pela existência de três incógnitas(relativas a cátions, ânions e solvente) ${ }^{40}$.

Como dito na introdução, a dinâmica de compensação de cargas em polímeros condutores eletrônicos vem sendo discutida em termos da utilização da $\mathrm{ELQ}^{7,8}$. Neste trabalho serão apresentados resultados do transporte iônico em eletrodos de ouro modificados com filmes de polirrol (PPY). Particularmente serão mostrados resultados utilizando o polipirrol eletropolimerizado em condições potenciostáticas $(E=700 \mathrm{mV}) \mathrm{em}$ solução aquosa contendo $53 \mathrm{mM}$ PPY e $0,3 \mathrm{M} \mathrm{LiClO}_{4}$. Os filmes foram depositados sobre Au até se obter uma massa de $40 \pm 2 \mu \mathrm{g} \mathrm{cm}^{-2}$ e uma espessura em torno de $0,1 \mu \mathrm{m}$. Os experimentos foram realizados em uma solução de $0,1 \mathrm{M}$ de $\mathrm{LiClO}_{4}$ em metanol com velocidade de varredura de $50 \mathrm{mV} \mathrm{s}^{-1}$. Detalhes sobre a realização dos experimentos e o comportamento redox do polipirrol em vários solventes podem ser encontrados em Varela et $a .^{43}$.

Na Figura 7a são mostrados os perfis de corrente e variação de massa (calculada a partir das variações de freqüência nos experimentos de MECQ utilizando a equação de Sauerbrey ${ }^{44}$ ). O perfil voltamétrico da Figura 7a ilustra um pico de oxidação/redução à $-0,2 /-0,4 \mathrm{~V}$ durante a varredura positiva/negativa e a razão entre as cargas anódica e catódica é aproximadamente igual a 1 . A variação de massa atinge um ganho de $3 \mu \mathrm{g} \mathrm{cm}^{-2}$ no final da varredura anódica e retorna a um valor consideravelmente próximo ao inicial na varredura reversa (catódica), esse aumento/diminuição de massa durante a oxidação/redução do filme reflete o processo de inserção/expulsão dos íons $\mathrm{ClO}_{4}^{-}$em base mássica ${ }^{45}$, já que esses íons apresentam massa molar bem superior aos íons $\mathrm{Li}^{+}$.

Através das equações (10) e (11) mostradas anteriormente, foram calculados os fluxos de cátions e ânions mais a participação do solvente, e os resultados são mostrados em função do potencial na Figura 7b. Uma importante constatação a partir dessa figura é a ausência de inversão de sinal nos fluxos de íons mais solvente (que, caso presente, indicaria a participação massiva de moléculas de solvente durante o processo de compensação de cargas). Assim, uma hipótese plausível seria que a participação de solvente poderia ser desconsiderada e os fluxos mostrados corresponderiam, sem prejuízo, às contribuiões de cátions e ânions. A participação de cátions e ânions durante o processo de eletroneutralização do polipirrol foi descrita por Kaufman et $a l .{ }^{46}$, no primeiro estudo do processo de compensação de cargas em eletrodos modificados com polipirrol, utilizando a técnica de MECQ. De fato, essa contribuição foi estimada como sendo em torno de $30 \%$ no presente trabalho ${ }^{43}$ e ambos os fluxos (cátions e ânions) atingem um máximo próximo de $-0,2 \mathrm{~V}$ como mostrado na Figura 7a.

Utilizando o mesmo procedimento descrito previamente ${ }^{8}$, os gráficos de dq/dt (=j) em função de q durante os processos de oxidação e redução, são mostrados nas Figuras $8 \mathrm{a}$ e $8 \mathrm{~b}$, respectivamente. Através da comparação com as curvas representando a utilização da ELQ, observa-se claramente que a relação entre corrente e carga não é descrita pela ELQ. De fato, experimentos com polipirrol em solução aquosa $1 \mathrm{M}$ de $\mathrm{NaCl}$ a $100 \mathrm{mV} / \mathrm{s}^{-1}$ levaram os autores a concluir que o processo não poderia ser satisfatoriamente descrito pela ELQ devido à ausência de picos bem definidos de oxidação e redução, no entanto, ambos picos são bem definidos no presente trabalho, como mostrado na Figura 7a. Isto pode ser considerado como conseqüência de que as propriedades de eletrodos poliméricos modificados dependem fortemente das condições de síntese e do meio utiliza$\operatorname{dos}^{14,40,47}$. Desvios em relação à curva dq/dt $(=\mathrm{j})$ em função de q durante os processos de oxidação e redução na região de maior carregamento também foram encontrados por Malek et al. ${ }^{9}$ em filmes 

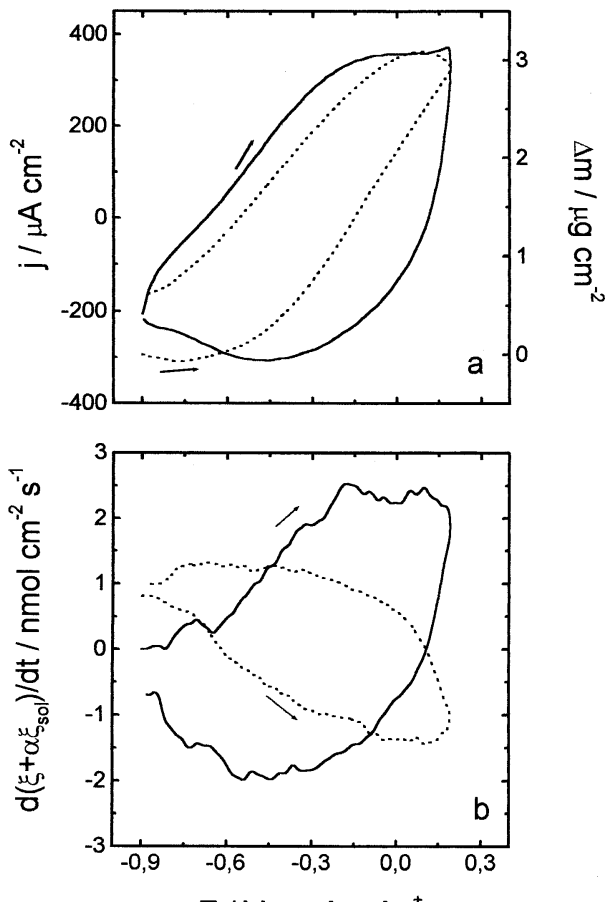

$\mathrm{E} / \mathrm{V}$ vs. $\mathrm{Ag}, \mathrm{Ag}^{+}$

Figura 7. Comportamento de filmes finos de polipirrol. (a) Dependência da corrente (linha pontilhada) e da variação de massa (linha contínua) com o potencial e (b) dependência dos fluxos de ânions (linha contínua) e cátions (linha pontilhada) (incluíndo o solvente) com o potencial.

finos de poli-orto-aminofenol na presença de diferentes eletrólitos e atribuídos à presença de regiões menos disponíveis no interior do filme para a acomodação de ânions. Tal discussão foi apoiada por resultados prévios tratando da participação de ânions através da dimensão fractal do filme polimérico ${ }^{48}$, no entanto, devido a ausência de medidas de MECQ, nada foi discutido a respeito de eventual participação de cátions no processo de compensação de cargas.

A partir da análise desse aparente desacordo entre os dados experimentais e o comportamento descrito pela ELQ, o processo de compensação de cargas pode ser investigado mais detalhadamente. Uma característica do voltamograma cíclico mostrado na Figura 7a a ser ressaltada é a componente capacitiva da corrente relacionada aos íons localizados mais externamente em relação à cadeia polimérica como discutido previamente por Tanguy et al ${ }^{49-51}$. Tal tratamento surgiu a partir da observação do efeito capacitivo que polímeros condutores eletrônicos (PCE) apresentam durante seu processo redox ${ }^{52,53}$. Assim, de acordo com esse modelo, íons localizados mais externamente em relação ao filme não estariam sujeitos às restrições de transporte descritas pela ELQ. Tal fato pode ser observado na Figura 7a na qual o decréscimo de corrente após um valor máximo não é observado já que parte dos íons responsáveis pela eletroneutralização do filme (condição necessária para que aconteça a transferência eletrônica na interface filme/metal) não sofre as restrições de transporte discutidas.

Prosseguindo na análise do transporte das espécies no curso do processo de eletroneutralização, as Figuras 9a e 9b ilustram os resultados obtidos para o fluxo de variação massa $(\mathrm{d}(\Delta \mathrm{m}) / \mathrm{dt})$ em função da variação de massa $(\Delta \mathrm{m})$ para os processos de oxidação e redução, respectivamente. Nesse caso observa-se claramente a melhor correlação entre os resultados experimentais e a ELQ, pelo menos em comparação aos resultados mostrados na Figura 8 para o fluxo de carga. Tal comportamento deve ser discutido em termos da diferença entre as massas molares dos íons que compensam a carga, ou seja, $\mathrm{ClO}_{4}^{-} \mathrm{e} \mathrm{Li}^{+}$, com massas molares de 99 e $7 \mathrm{~g} \mathrm{~mol}^{-1}$, respectivamente.

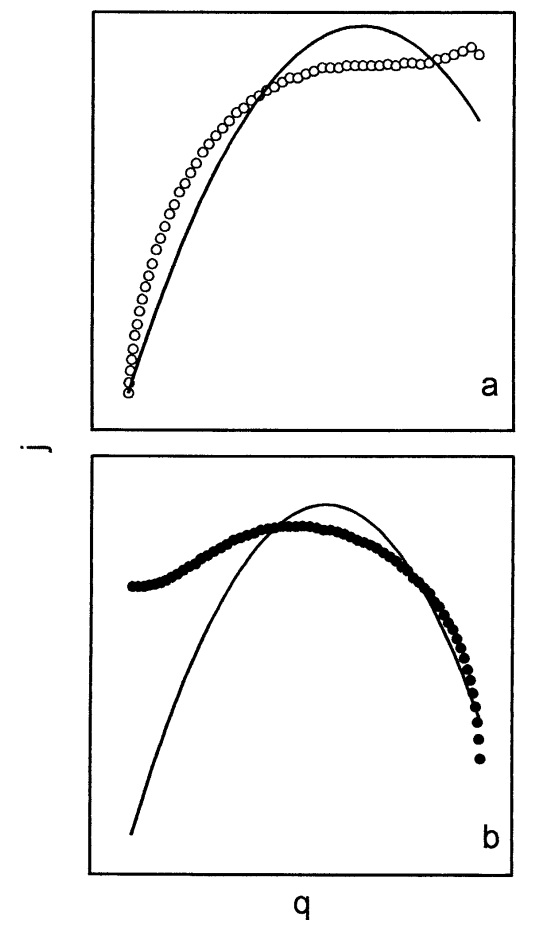

Figura 8. Comportamento de filmes finos de polipirrol. (a) comparação da dependência da corrente $(j=d q / d t)$ com a carga (normalizadas) para o processo de oxidação com a forma normalizada da ELQ. (b) Idem para o processo de redução.

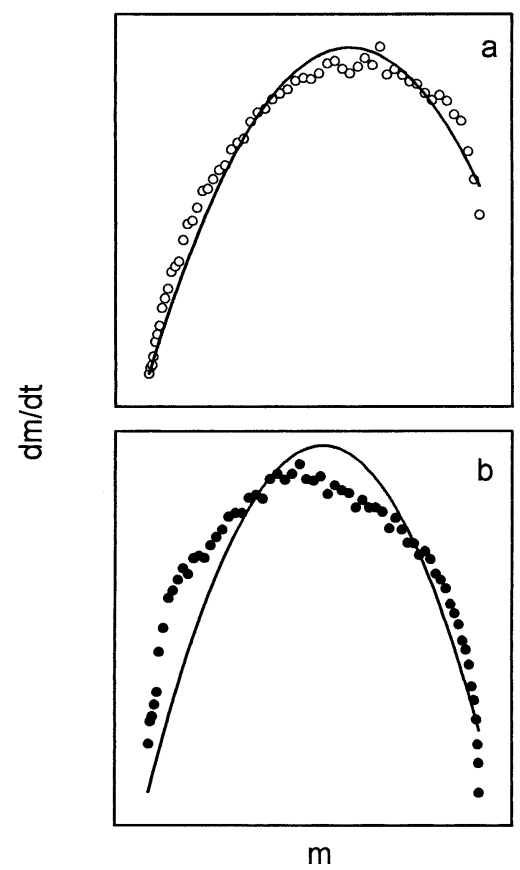

Figura 9. Comportamento de filmes finos de polipirrol. Dependência do fluxo de variação de massa com a variação de massa (normalizados) para os processos de (a) oxidação e (b) redução em comparação com a forma normalizada da ELQ.

Assim, a resposta em termos de massa é dominada pela contribuição dos ânions como mostrado no perfil de variação de massa (Figura 7a) representando majoritariamente a inserção/expulsão de ânions durante a oxidação/redução como discutido. 
Através das correlações obtidas para os fluxos de carga e massa mostrados anteriormente pode-se inferir sobre a natureza dos íons responsáveis pelos desvios em relação à ELQ observados nos fluxos de carga para ambos os processos de oxidação e redução mostrados na Figura 8. Como o fluxo de massa é dominado pelo transporte de ânions e o comportamento desse fluxo é bem descrito pela ELQ, parece intuitivo supor que o transporte de cátions afeta a resposta de corrente observada, porque esse transporte não estaria sujeito aos fenômenos descritos pela ELQ, já que os cátions estariam localizados mais externamente em relação ao filme e os efeitos de repulsão (responsáveis pela re-alimentação negativa) são minimizados. Por outro lado os ânions estariam localizados mais internamente na cadeia compensando a carga positiva dos sítios criados durante a oxidação do filme e seu transporte seria bem descrito pela ELQ.

Através das equações (10) e (11) mostradas anteriormente, foram calculados os fluxos de cátions e ânions incluindo as contribuições do solvente. Os resultados são mostrados na Figura 10, na qual esses fluxos são graficados em função dos números de mols. Em ambos os processos de oxidação e redução, são obtidas boas correlações no caso dos transporte de ânions, enquanto que as discrepâncias entre os resultados experimentais e a ELQ são evidentes para o transporte de cátions.

\section{DISCUSSÃO}

Como todo avanço no entendimento de fenômenos conhecidos através de conceitos modernos, a utilização da ELQ em sistemas eletroquímicos sofreu algumas críticas e, após a publicação dos primeiros trabalhos devotados à sua utilização na descrição de procesos eletroquímicos ${ }^{8}$, algumas discussões foram também publicadas sobre a validade de tal analogia e análise ${ }^{54,55}$. Os principais pontos dessa discussão já foram convenientemente elucidados ${ }^{56-59}$ e aqui será abordada especialmente a interpretação do processo de compensação de cargas em termos da utilização de isotermas de adsorção como uma generalização do processo de intercalação em matrizes inorgânicas (ver Levi et al. ${ }^{60}$ e referências inseridas para uma revisão geral sobre esse tema).

Em termos formais, o processo de intercalação pode ser definido como a acomodação de espécies "convidadas" em uma matriz sólida do material hospedeiro ${ }^{61}$. Dois aspectos relacionados às espécies convidada e hospedeira são característicos do processo de intercalação: (a) espécies convidadas ocupam sítios específicos na matriz hospedeira, o número máximo acessível de sítios é fixo, e a fração de sítios ocupados pode variar em função do potencial do eletrodo, e (b) as espécies convidadas se deslocam dentro da matriz de um sítio para outro. Como definido, pode-se afirmar que o processo de intercalação pode ser diretamente relacionado com o processo de adsorção de espécies em uma interface e ambos os processos (intercalação e adsorção) podem ser descritos por isotermas de adsorção. No entanto, algumas considerações devem ser feitas.

Durante o processo de intercalação, a eletroneutralidade local no seio da matriz hospederira implica no fato de que um número equivalente de elétrons transferidos do substrato metálico deve estar localizado nos sítios ocupados pelos íons intercalados. Assim, há uma importante diferença a ser ressaltada entre os processos de intercalação e adsorção. As condições de equilíbrio para o processo de intercalação incluem a distribuição de ambas as contribuições, iônica e eletrônica, dentro da matriz hospedeira, enquanto o processo de adsorção está relacionado exclusivamente às espécies iônicas em solução (obviamente desconsiderando o caso de transferência parcial de carga durante a adsorção). Outro fato a ser discutido é sobre a perturbação imposta ao sistema durante o processo de intercalação. De fato, diferentes escalas de tempo resultam na inclusão de outros
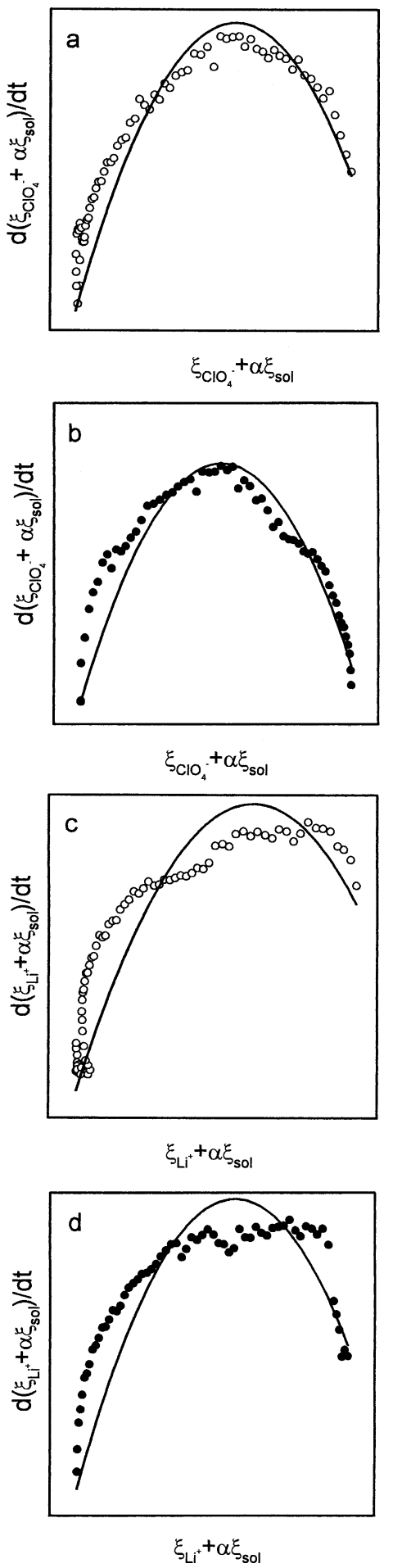

Figura 10. Comportamento de filmes finos de polipirrol. Dependência do fluxo de ânions (e solvente) com o respectivo número de mols (normalizados) para os processos de (a) oxidação e (b) redução em comparação com a forma normalizada da ELQ. As figuras (c) e (d) mostram os correspondentes processos para o fluxo de cátions (e solvente).

processos (como transferência de massa, por exemplo) a serem considerados. Aplicações envolvendo isotermas, como a isoterma de Frumkin, na descrição de processos de intercalação utilizam velocidades de varredura da ordem de dezenas de $\mu \mathrm{Vs}^{-1}$, bem menores que as velocidades de varredura geralmente utilizadas nos experimentos descritos, da ordem de algumas dezenas de $\mathrm{mVs}^{-1}$. 
Outro problema associado à utilização de isotermas de adsorção na interpretação de processos descritos aqui como de intercalação reside nas diferenças entre as matrizes orgânicas e inorgâncias com respeito às espécies intercalantes. Em contraste com matrizes inorgânicas, sistemas mais complexos como no caso de polímeros condutores eletrônicos (PCE's), onde se observa a participação de cátions, ânions e eventuais moléculas de solvente, a intercalação é melhor referida como processo de compensação de cargas ou de eletroneutralização e considerações adicionais devem ser levadas em conta na análise desses sistemas. De fato, diferentemente dos materiais inorgânicos, PCE's são caracterizados por uma estrutura macromolecular flexível que se manifesta em função das mudanças conformacionais, inchamento por entrada de solvente e interações mecânicas e eletrônicas entre contra-íons e cargas elétricas dos sítios da cadeia polimérica durante o processo de dopagem ${ }^{62}$ e algumas hipóteses requeridas para a abordagem através do tratamento baseado nas isotermas de adsorção devem ser consideradas.

Por outro lado, a utilização da ELQ em detrimento às isotermas de adsorção nos sistemas ilustrados (em particular no processo de eletroneutralização em PCE's) se apresenta como mais adequada, principalmente devido aos conceitos relacionados à população iônica no interior da matriz hospederia.

\section{CONSIDERAÇÕES FINAIS}

A utilização da ELQ na sua forma discreta em teoria de caos tem revelado a riqueza do conteúdo conceitual desta equação. Entretanto, apesar de tal riqueza conceitual, pode-se afirmar que os aspectos fundamentais da forma diferencial da ELQ têm sido pouco explorados. Este trabalho mostra os fundamentos dessa equação, que pode ser considerada uma ferramenta de grande utilidade em processos sujeitos a dissipação e re-alimentação. Isto foi ilustrado aqui para alguns sistemas eletroquímicos, onde o fluxo de cargas é, tipicamente, um processo dissipativo e re-alimentado. É de se esperar que o tratamento e os exemplos aqui apresentados estimulem uma aplicação mais generalizada da ELQ, tanto na físico-química como em outras áreas do conhecimento.

\section{AGRADECIMENTOS}

Os autores agradecem os auxílios financeiros recebidos da FAPESP, CNPq e FINEP (PRONEX).

\section{REFERÊNCIAS}

1. Malthus, T. R.; An Essay on the Principle of Population; Pickering; London, 1986.

2. Sidl, I.; Tisdell, C. A.; Ecol. Econ. 1999, 31, 395.

3. Verhulst, P. F.; Correspondance Math. Phys. 1838, 10, 113.

4. Edelstein-Keshet, L.; Mathematical Models in Biology; Random House, New York, 1988.

5. Gonzalez, E. R.; J. Electrochem. Soc. 1996, 143, L113.

6. De Sena, D. R.; Ticianelli, E. A.; Gonzalez, E. R.; Electrochim. Acta 1998, $43,3755$.

7. Córdoba de Torresi, S. I.; Torresi, R. M.; Gonzalez, E. R.; Synth. Met. 1999, 101,800 .

8. Torresi, R. M.; Córdoba de Torresi, S. I.; Gonzalez, E. R.; J. Electroanal. Chem. 1999, 461, 161.

9. Malek, K.; Gobal, F.; Synth. Met. 2000, 113, 167.

10. Senda, M.; Yoshida, N.; Shimakawa, K.; Philosop. Mag. Lett. 1999, 79, 375 .

11. Zoppi, R. A.; De Paoli, M. A.; Quim. Nova 1993, 16, 560.

12. De Paoli, M. A.; Panero, S.; Paserini, S.; Scrosati, B.; Adv. Mat. 1990, 2, 480 .

13. Oliveira, S. C.; Torresi, R. M.; De Torresi, S. I. C.; Quim. Nova 2000, 23, 79.
14. Mattoso, L. H. C.; Quim. Nova 1996, 19, 388.

15. Monk, P. M. S.; Mortinimer, R. J.; Rosseinsky, D. R.; Electrochromism: Fundamentals and Applications, VCH, Weinheim, 1995.

16. Novák, P.; Müler, K.; Santhanam, K. S. V.; Haas, O.; Chem. Rev. 1997, 97, 207

17. Strogatz, S. H.; Nonlinear Dynamics and Chaos; Addison-Wesley Pub.; New York, 1994.

18. Hilborn, R. C.; Chaos and Nonlinear Dynamics, Oxford University Press, Oxford, 1994.

19. Scott, S. K.; Chemical Chaos, Clarendon Press, Oxford, 1991

20. Nicolis, G.; Introduction to Nonlinear Science, Cambridge University Press, Cambridge, 1995.

21. Murray, J. D.; Mathematical Biology; Springer, $2^{\text {nd }}$ Edition, Seattle, 1991.

22. Peleg, M.; Crit. Rev. Food Sci. 1997, 37, 471.

23. Huzimura, R.; Matsuyama, T.; Theor. Popul. Biol. 1999, 56, 301.

24. Wan, X. R.; Wang, M. J.; Wang, G. H.; Zhong, W. Q.; Acta Theriol. 2000, 45,145

25. Luo, Y.; Epstein, I.; Adv. Chem. Phys. 1991, 79, 269.

26. Lotka, A. J.; J. Am. Chem. Soc. 1920, 42, 1595.

27. Volterra, V.; Nature 1926, 118, 558.

28. Nicolis, G.; Prigogine, Y.; Self-Organization in Nonequilibrium Systems, John Wiley \& Sons, New York, 1977.

29. Epstein, I. R.; Pojman, J. A.; An Introduction to Nonlinear Chemical Dynamics, Oxford University Press Inc., New York, 1998.

30. Nicolis, G.; Prigogine, Y.; Exploring Complexity: An introduction, W. H. Freeman and Company, New York, 1989, p. 217.

31. Gonsalves, M.; Hillman, A. R.; J. Electroanal. Chem. 1998, 454, 183.

32. Torresi, S. I. C. de; Electrochim. Acta 1995, 40, 1101.

33. Torresi, S. I. C. de; Gabrielli, C.; Hugot Le Goff, A.; Torresi, R.; J. Electrochem. Soc. 1991, 138, 1548.

34. Scrosati, B.; Application of Electroactive Polymers, Chapman \& Hall, New York, 1993.

35. Girotto, E. M.; De Paoli, M. A.; Quim. Nova 1999, 22, 358.

36. Buttry, D.A.; In: Electroanalytical Chemistry, Bard, A., ed.; vol. 17, 1999, Marcel Dekker, New York.

37. Hepel, M.; In: Interfacial Electrochemistry, Wieckowiski, A., ed.; 1999, Marcel Dekker, New York.

38. Varela, H.; Malta, M.; Torresi, R. M.; Quim. Nova, 2000, 23, 664.

39. Lee, J.; Varela, H.; Uhm, S.; Tak, Y.; Electrochem. Commun. 2000, 2, 646.

40. Maia, G.; Torresi, R. M.; Ticianelli, E. A.; Nart, F. C.; J. Phys. Chem. 1996, 100,15910 .

41. Varela, H.; Torresi, R. M.; Buttry, D. A.; J. Braz. Chem. Soc. 2000, 11, 32.

42. Varela, H., Torresi, R.M.; J. Electrochem. Soc. 2000, 147, 665.

43. Varela, H.; Malta, M.; Torresi, R. M; J. Power Sources 2001, 92, 50.

44. Sauerbrey, G.; Z. Phys. 1964, 178, 457.

45. Varela, H.; Torresi, R. M.; Buttry, D. A.; J. Electrochem. Soc. 2000, 147, 4217.

46. Kaufman, J. H.; Kanazawa, K. K.; Street, G. B.; Phys. Rev. Lett. 1984, 53, 2461.

47. Giacomini, M. T.; Ticianelli, E. A.; Quim. Nova 1999, 22, 639.

48. Gobal, F.; Malek, K.; Mahjani, M. G.; Jafarian, M.; Safarnavadeh, V.; Synth Met. 2000, 108, 15.

49. Tanguy, J.; Mermilliod, N.; Hoclet, M.; Synth Met. 1987, 18, 7.

50. Mermilliod, N.; Tanguy, J.; Hoclet, M.; Sayed, A. A.; Synth Met. 1987, 18 359.

51. Tanguy, J.; Mermilliod, N.; Hoclet, M.; J. Electrochem. Soc. 1987, 134, 795.

52. Kobayashi, T.; Yoneyama, H.; Tamura, H.; J. Electroanal. Chem. 1984, 177, 281

53. Waltman, R. J.; Bargon, J.; Diaz, A. F.; J. Phys. Chem. 1983, 87, 1459.

54. Diard, J. P.; Gorrec, B. Le; Montella, C.; J. Electroanal. Chem. 1999, 475, 190.

55. Sadkowski, A.; J. Electroanal. Chem. 2000, 486, 92.

56. Torresi, R. M.; Córdoba de Torresi, S. I.; Gonzalez, E. R., J. Electroanal. Chem. 1999, 475, 193.

57. Torresi, R. M.; Córdoba de Torresi, S. I.; Gonzalez, E. R., J. Electroanal. Chem. 2000, 486, 95.

58. Diard, J. P.; Le Gorrec, B.; Montella, C.; J. Electroanal. Chem. 2000, 486, 97.

59. Sadkowski, A.; J. Electroanal. Chem. 2000, 486, 98.

60. Levi, M. D.; Aurbach, D.; Electrochim. Acta 1999, 45, 167.

61. McKinnon, W. R.; In: Solid-State Electrochemistry, Bruce, P. G., ed.;1995, p. 163, Cambridge University Press.

62. Levi, M. D.; Lopez, C.; Vieil, E.; Vorotyntsev, M. A.; Electrochim. Acta 1997, 42, 757 